\title{
A robust mesh optimisation method for multiphase porous media flows
}

\author{
P. Salinas ${ }^{1}$ (D) . D. Pavlidis ${ }^{1} \cdot$ Z. Xie $^{1} \cdot$ H. Osman ${ }^{1}$. C. C. Pain ${ }^{1}$ - M. D. Jackson ${ }^{1}$
}

Received: 8 June 2017 / Accepted: 11 July 2018 / Published online: 30 July 2018

(C) The Author(s) 2018

\begin{abstract}
Flows of multiple fluid phases are common in many subsurface reservoirs. Numerical simulation of these flows can be challenging and computationally expensive. Dynamic adaptive mesh optimisation and related approaches, such as adaptive grid refinement can increase solution accuracy at reduced computational cost. However, in models or parts of the model domain, where the local Courant number is large, the solution may propagate beyond the region in which the mesh is refined, resulting in reduced solution accuracy, which can never be recovered. A methodology is presented here to modify the mesh within the non-linear solver. The method allows efficient application of dynamic mesh adaptivity techniques even with high Courant numbers. These high Courant numbers may not be desired but a consequence of the heterogeneity of the domain. Therefore, the method presented can be considered as a more robust and accurate version of the standard dynamic mesh adaptivity techniques.
\end{abstract}

Keywords Mesh adaptivity $\cdot$ Multi-phase flows $\cdot$ Porous media flows

\section{Introduction}

Multiphase porous media flow is important in many subsurface reservoirs, including hydrocarbon reservoirs ([11]), unconfined aquifers ([25]), targets for geological CO2 storage ([45]) and magma reservoirs ([44]). Such reservoirs are typically heterogeneous, with material properties, such as permeability varying over orders of magnitude. Moreover, the spatial arrangement of heterogeneity is often complex, governed by geological features, such as faults, fractures and contrasting rock types or lithologies (e.g. [7]). Numerical simulation of multiphase flow in these reservoirs is often challenging and computationally expensive, because of the complex spatial arrangement and wide variability in material properties. Moreover, solutions to the governing flow equations often include large changes in solution variables, such as saturation, composition or flow velocity over short length-scales (for example, at displacement fronts when one

P. Salinas

pablo.salinas@imperial.ac.uk

www.imperial.ac.uk/earth-science/research/research-groups/norms/

1 Novel Reservoir Modelling and Simulation Group, Department of Earth Science and Engineering, Imperial College London, London, UK fluid displaces another) and local effects related to heterogeneity or boundary conditions (for example, coning around boreholes). Resolving these features requires high mesh resolution, but this significantly increases computational cost in a large model domain.

A number of studies have proposed the use of mesh adaptivity methods to maintain or increase solution accuracy at lower computational cost. Such methods include adaptive mesh refinement (AMR; [10, 15, 16, 23, 27, 42]) and dynamic mesh optimisation (DMO; [22, 24, 32, 48]). In these methods, the mesh is modified during the simulation to increase solution quality based on some form of metric extracted from the solution field(s) of interest. Mesh adaptivity is commonly applied in other areas of computational fluid dynamics ([4]), although its application in porous media flow is less widespread.

The complex geometries found in many subsurface reservoirs lead to a wide range of mesh element sizes in discretised reservoir models $([13,21])$. Moreover, the flow velocities in these models can vary significantly, reflecting the large variations in material properties. Consequently, the local Courant number can take a broad range of time varying values and may be large in some parts of the model domain. The non-linear solvers used in multiphase porous media flow simulations can handle such large Courant numbers $([5,26,28,39])$. Moreover, numerical simulations 
with local Courant numbers greater than unity can yield satisfactory solutions $([33,39])$. However, the successful application of mesh adaptivity with large local Courant numbers is not guaranteed. The most limiting factor of mesh adaptivity for porous media flow has been the interpolation of the petrophysical properties across meshes. In conventional reservoir-simulation models, initially geologic heterogenenity was neglected $([9,43])$. Later on the material properties were re-scaled onto the fine grid ([23, 47]). Nonetheless, the computational effort required for this is very high and outweighs the advantages of mesh refinements. Another different approach to modelling multiphase flow is the use of surfaces to represent geological heterogeneities $([12,24,29,38])$. Here, the petrophysical properties are constant within a surface, and therefore there is no need to interpolate them between meshes as long as the surfaces are kept. Recently, [36] has analysed the number of surfaces required to model realistic reservoirs, showing that a very reduced number of surfaces (around 50) is required to obtain very accurate results.

The use of mesh adaptivity in time-dependent problems implies that the mesh is always one step behind the solution if it is adapting to the solution at the current time level (e.g. [3]). If the Courant number is below or equal to unity this is not problematic, as the mesh can be (re-) adapted before the solution has moved into coarser regions of the mesh. However, if the local Courant number is very large, the solution accuracy may be compromised because the solution moves into coarser regions of the mesh, and therefore, represented at lower resolution. Once lost, the high resolution aspects of the solution can never be recovered by mesh adaptivity, as the metric used to refine the mesh is based upon the last obtained solution (e.g. Fig. 1). This is problematic for multiphase porous media flow as the Courant number used in this field are usually larger than in other CFD areas. Many methods have been proposed

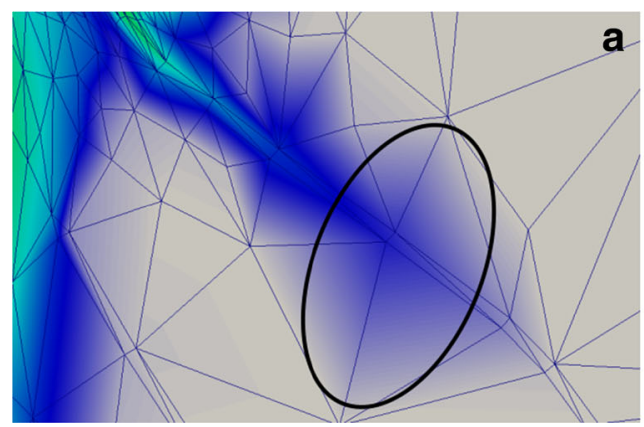

Fig. 1 Mesh adaptivity failure with large Courant number. a Solution field before the mesh is adapted to the field. The solution has reached a low resolution region of the mesh, which introduces numerical diffusion, unphysically altering the result. b Solution field after the mesh to address this problem. The most simple one is to use mesh gradation, which ensures that the mesh resolution does not vary significantly between neighbouring elements, and therefore, this expands the high resolution area to create a "safety net". This can be used to reduce the frequency of adapts or to deal with higher Courant numbers. Another, method develop is named metric advection, here the mesh is transported with the flow using the current flow velocities [22]. Improved results have been obtained for Courant numbers of up to 5 . These techniques are very useful for Courant numbers of up to tenths, enabling to use mesh adaptivity for Courant numbers above unity and/or adapt less frequently. However, these techniques are not useful for very high Courant numbers like the ones studied here. Mesh gradation for very high Courant numbers requires massive areas of high resolution to ensure that the next time-level is properly captured. On the other hand, metric advection for multiphase porous media flow is limited as the velocity fields are not divergent-free within each phase, so the phase velocity is not necessarily a good indicator of where, for example, displacement fronts (and thus where fine mesh resolution is required) will end up at the end of a large time-step or series of time-steps.

Alauzet et al. [3] presented a modification of the usual procedure of mesh adaptivity for Navier-Stokes flow, in which the mesh is adapted as part of the non-linear solver to allow use of the most recently updated solution field. The method proposed here builds on this concept but it is tailored for multiphase porous media flows.

The paper is organised as follows. A summary of the governing equations used in the numerical experiments is presented in Section 2. In Section 3, a summary of standard DMO is presented, followed by a description of the proposed method. Section 4 reports some numerical results showing the performance of the proposed method. Finally, some concluding remarks are presented in Section 5. 


\section{Governing equations}

Here, we present a summary of the discretisation technique used in the numerical experiments reported here. However, the new mesh optimisation method is independent of the discretisation technique used. The spatial discretisation is based on the control volume finite element method (see $[18-20,24,30,31]$ ), with a variation introduced in [40] to increase the robustness of the method against large-angle elements. Here, triangular meshes (2D) or tetrahedra (3D) are used to mesh the domain. Control volumes are created around the vertexes of the elements by connecting the barycentres of the elements with the midpoint of the edges (see Fig. 2). In this paper, the element pair $P_{0} \mathrm{DGP}_{1}(\mathrm{CV})$ is used for the numerical simulations. The velocity is discretised using finite elements, using discontinuous Galerkin elements and with one degree less $\left(P_{0} \mathrm{DG}\right)$ than the pressure, which is discretised using CVs $\left(P_{1}(\mathrm{CV})\right)$. The petrophysical properties are defined element wise, while the saturation is defined CV-wise (Fig. 2). For the time discretisation, a $\Theta$-method is used. $\Theta$ smoothly varies between 0.5 (Crank-Nicholson) and 1 (implicit Euler) based on a total variation diminishing (TVD) criterion. The non-linear solver used here is a modification of the Anderson fixed-point solver detailed in [39]. The presented numerical methods reported here are implemented in the open-source code IC-FERST (Imperial College Finite Element Reservoir SimulaTor).

Darcy's law can be written as follows:

$\mu_{\alpha} S_{\alpha}\left(\mathcal{K}_{r_{\alpha}} K\right)^{-1} u_{\alpha}=-\nabla p+s_{u \alpha}$,

in which $p$ is the pressure, $u_{\alpha}$ is the phase saturationweighted Darcy velocity of phase $\alpha$, which relates to the Darcy velocity as $q_{\alpha}=\frac{u_{\alpha}}{S_{\alpha}}$. This velocity is used to try to reduce the non-linearity introduced through the relative permeability term ([20]). $s_{u \alpha}$ is a source term, $K$ is the permeability tensor, $\mathcal{K}_{r_{\alpha}}, \mu_{\alpha}$ and $S_{\alpha}$ are the relative permeability, viscosity and saturation of phase $\alpha$ respectively. For simplicity, two phase immiscible flow is considered here with no gravity forces nor capillary forces and rock and fluids are considered incompressible.

The saturation equation, can be written as follows:

$\phi \frac{\partial S_{\alpha}}{\partial t}+\nabla \cdot\left(u_{\alpha} S_{\alpha}\right)=s_{c t y, \alpha}$,

where $\phi$ is the porosity and $s_{c t y, \alpha}$ is a source term.

To close the system of equations, the saturation is constrained by:

$\sum_{\alpha=1}^{n} S_{\alpha}=1, \quad \forall n$,

where $n$ is the number of phases.

\section{Mesh adaptivity for porous media flow}

\subsection{Dynamic mesh optimisation summary}

The approach utilises the anisotropic mesh optimisation library of [37], in which mesh elements may be split or amalgamated, elements edges may be swapped, or element vertices may be moved. A brief summary is detailed here. The DMO routine used here starts by estimating the $L_{2}$ norm of the local interpolation error introduced by the FE representation, using the weighted norm of the Hessian matrix of the specified fields $H$. If the variables are defined into the $\mathrm{CV}$ space, they are projected into the FE. The Hessian of a smooth field can be approximated by a Galerkin projection of the linear interpolation on the mesh ([4]). This yields the estimation of the interpolation error of the field based on information already available on the mesh. A functional defining the quality of the mesh is then created:

$$
I=\sum_{i \in \text { edges }}\left(e_{i}^{t} E e_{i}-1\right)^{2}
$$

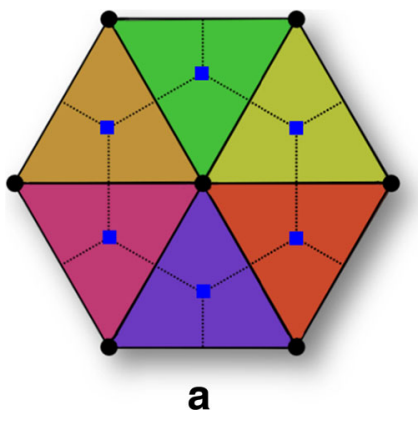

Fig. 2 Example of a $P_{0} \mathrm{DGP}_{1}(\mathrm{CV})$ element pair, a shows the elements with different colours and the $\mathrm{CVs}$ with dotted lines. The petrophysical properties are defined in this mesh; the velocity is constant within an

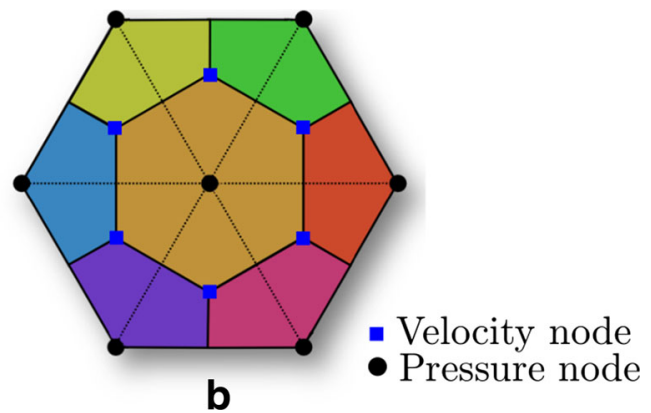

element. b Shows the CVs with different colours and the elements with dotted lines. The saturation is discretised in this mesh; the pressure is defined at the centre of each $\mathrm{CV}$ 
in which $e_{i}$ define the edges connecting the vertices of the mesh and

$E=\operatorname{det}(H)^{\frac{1}{2 \gamma \delta}} \frac{|H|}{\epsilon}$

defines the estimation of the error metric along each edge. Here, $\epsilon$ specifies the required tolerance for the field introduced by the user, $\gamma$ is the degree of the polynomial of the norm used and $\delta$ is the number of dimensions of the problem. The generation of the mesh now consists in creating a mesh with an approximately uniform interpolation error and fulfilling other criteria introduced by the user, such as geological surfaces, number of elements, mesh anisotropy, mesh gradation level, etc. Therefore, the old mesh is modified in order to obtain a new mesh using the following techniques:

- Refining by splitting: adding extra node in an element.

- Coarsening by edge collapse: replacing two vertices by a single one.

- Face-edge and edge-face swap: reordering the connectivity of existing elements.

- Node movement: repositioning of a vertex.

Modifications to the current mesh/element are accepted only in the change improves the metric criterion defined. Otherwise, the mesh/element is not modified. Once the new mesh has been generated the fields from the old mesh are interpolated to the new mesh, many techniques are available for this ([2]).

\subsection{Dynamic mesh optimisation for porous media flow}

In the numerical experiments reported here, DMO is used to capture variations in saturation, pressure and other key solution fields of interest, increasing the mesh resolution where required and coarsening elsewhere. A surface-based representation of geologic heterogeneity is used ([24]). Surfaces are honour by the adaptivity routine by generating some "master" nodes that cannot be removed or modified by the mesh adaptivity routine. Nonetheless, more nodes can be added if required to improve the solution metric of interest (Eq. 5).

In this paper, the pressure and velocity interpolation uses a conservative Galerkin technique ([17]), while the saturation interpolation used a CV-Galerkin interpolation, which is fully conservative and bounded and has second order re-mapping ([2]). There is no need to recalculate values of material properties, such as porosity and permeability, because these are constant within each geologic domain and are simply assigned to mesh elements within the domain. Thus up-, cross- or downscaling of

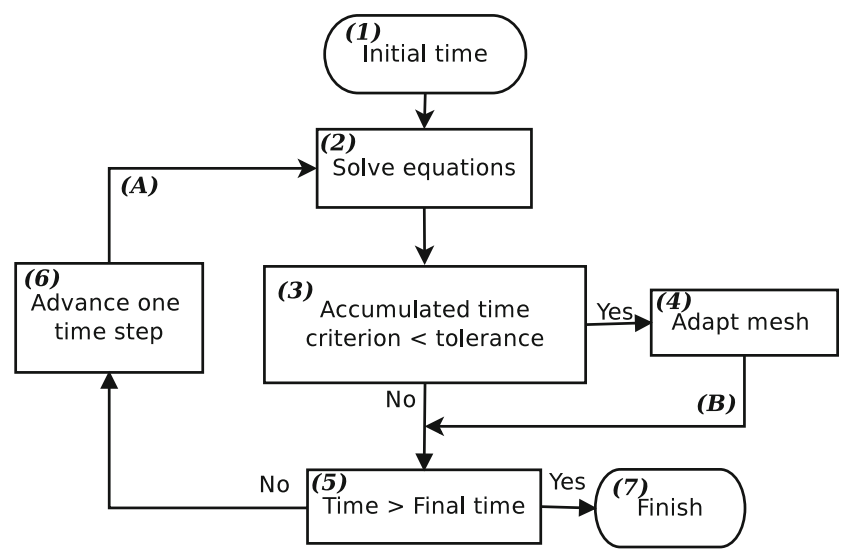

Fig. 3 Standard DMO in which the mesh is adapted after solving the equations over a number of time-steps

material properties during DMO is not required. Saturationdependent material properties, such as relative permeability are recalculated using the local saturation in each $\mathrm{CV}$, in similar manner to a conventional fixed-grid approach.

Figure 3 shows the standard methodology to adapt the mesh for porous media simulations. First, the equations are solved as usual (Fig. 3 box (2)). Next, the accumulated timesteps or accumulated time is checked (Fig. 3 box (3)). Based on this check, the algorithm may enter loop (B) Fig. 3, in which the mesh is adapted (Fig. 3 box (4)). Either way, if the final time has not been reached, the time is increased (Fig. 3 box (4)) and the process is repeated until the final time-level is reached or exceeded.

\subsection{Mesh optimisation for high Courant numbers}

The new method is based on two steps that are applied sequentially. Each step is described in the following sections and the overall algorithm is presented in Fig. 4. Compared

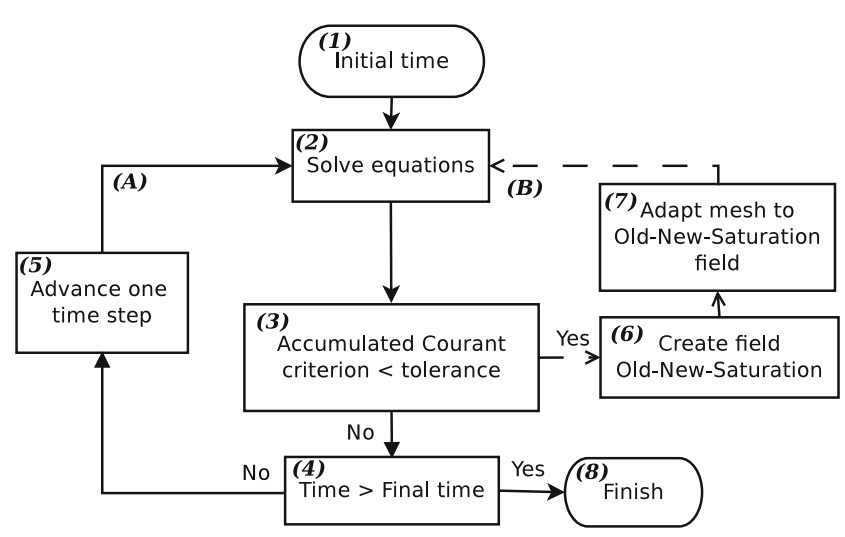

Fig. 4 Non-linear mesh adaptivity. The mesh is adapted as part of the non-linear procedure. Adding loop (B) to the usual method described in Fig. 3 
with the standard approach represented in Fig. 3, the new method differs in loop (B) Fig. 4. The new approach is implemented in the context of DMO, however as long as the mesh refinement is based on the same principles this approach can be used for AMR as well as DMO. Moreover, in the new approach, AMR/DMO is triggered based on an accumulated Courant number, i.e. when the solution has moved certain number of CVs.

We demonstrate the method using the specific example of a non-wetting phase being displaced by a wetting phase in a two-dimensional (2D) domain where the mesh adapts to the saturation field Fig. 5. However, the method is applicable to any single or multiphase porous media flow problem and to cases where the mesh adapts to different solution fields.

\subsubsection{Standard approach for solving the system}

Figure 5a shows a case with a high Courant number that forces the solution to move into a region that has a relatively coarse resolution. In this problem, numerical diffusion is therefore increased, removing the advantages of using AMR/DMO. In the new approach, the accumulated Courant number is tested and adaptivity is triggered when the solution has moved a number of CVs, being this number decided by the user, Fig. 4 box (3), and the algorithm enters loop (B) in Fig. 4.

In this first step of the new method, it is not necessary to achieve convergence with the same quality as required at the end of the time-level. The mesh will change, so the solution will also change. Consequently, the convergence criteria of the non-linear solver can be reduced in order to rapidly obtain a reasonable solution, in this case for the saturation field. For large time-step sizes, this feature of the method can be used to incrementally increase the mesh resolution during the iterative process so that much of the non-linear work is performed on coarser meshes. The convergence

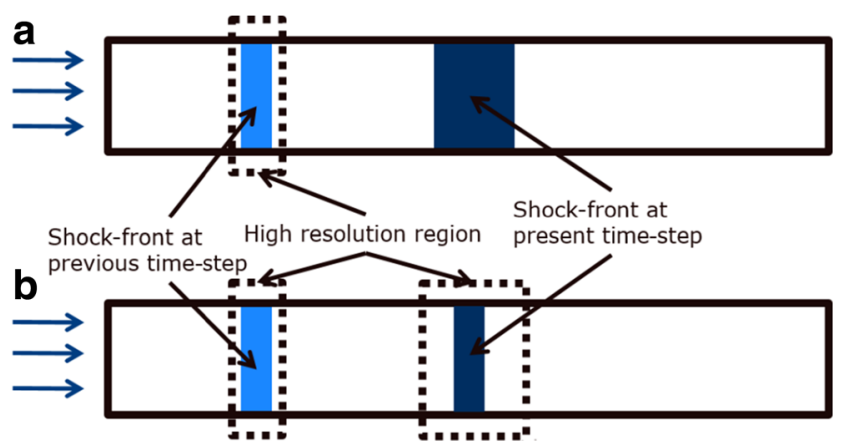

Fig. 5 The two steps to adapt the mesh within the non-linear solver. (Top) A solution is calculated as usual. (Bottom) The mesh is adapted considering the old and new saturation fields. The dashed lines denote regions of high resolution; the arrows represent the direction of the flow; the filled rectangles represents the positions of the displacement fronts in this problem at different time-levels criterion of the non-linear solver used in this paper is defined in terms of the saturation field as follows:

$f(S)=\sum_{i}^{N}\left(\frac{S_{\alpha}^{\prime}-S_{\alpha_{o}}}{S_{\alpha_{1}}-S_{\alpha_{o}}}\right)_{i}^{2} \frac{1}{N^{2}}$,

where $S_{\alpha}^{\prime}, S_{\alpha_{o}}$ and $S_{\alpha_{1}}$ are, respectively, the saturation of the latest non-linear solver iteration, the saturation at the previous non-linear iteration and the saturation after the first non-linear iteration at this current time-level. $N$ is the total number of control-volumes. Hence, the reduction of the difference of saturation is tested. This criterion has been used previously for non-linear solvers for multiphase porous media flow, and it requires fewer computations than calculating the residual ([46]). In this case, only the saturation is checked as the main source of non-linearity is controlled by saturation via saturation-dependent properties, such as relative permeability ([26]). In other problems, it may be necessary to test convergence using other solution fields. In this paper, a value of $f(S)=1 \times 10^{-1}$ is used to obtain the first approximation solution to which the mesh is adapted, and a value of $f(S)=5 \times 10^{-2}$ to achieve convergence before moving to the next time-level.

\subsubsection{Adapting to preserve old and new solution fields}

As shown in Eq. 2, to calculate the new solution, the solution from the previous time-level is required. Therefore, in order to adapt the mesh within the non-linear solver and obtain a solution on the new mesh by the end of the current timelevel, it is necessary to have high resolution solutions at both the previous and current time-levels. If the resolution of the solution at the previous time-level is not preserved, the loss of solution quality is then propagated to the next time-level.

A new solution field is created that is used to guide mesh adaptivity (Eq. 5), Fig. 4 box (7). This field is calculated here using:

$S_{\mathrm{comb}}=\operatorname{abs}\left(S_{w}^{t}-S_{w}^{t-1}\right)^{0.8}$

where $S_{w}^{t}$ and $S_{w}^{t-1}$ are the saturation of the wetting phase in the current and previous time levels, respectively. This approach is able to correctly represent the solution at both time-levels. However, other methods could be used so long as the new solution field is able to properly guide the DMO algorithm.

After the field is created, the mesh is adapted to the new field, Fig. 4 box (7) and Fig. 5b, interpolating to the new mesh all the necessary fields to continue the non-linear solver at that time-level with the new mesh. A reasonable estimation of the final solution is now available (the saturation obtained in the previous guess), so only a few iterations are required to achieve convergence. 
An optional extra step is to adapt the mesh again after obtaining the solution at the current time-level in order to reduce the number of elements. However, we have not seen this to be an improvement, as the reduction in the number of elements tends to be small and may not compensate for the extra cost of creating a new mesh and interpolating all the fields.

\section{Numerical experiments}

The methodology described in Fig. 5 is tested using three different cases of two-phase immiscible flow, in which an injected wetting phase displaces a non-wetting phase in a porous medium. The challenging part of the solution to capture in these examples is the sharp displacement (shock) front between the displacing and displaced phases. In all cases, the relative permeability is calculated using the Brooks-Corey correlation, [8]:

$$
\begin{aligned}
& k_{r w}\left(S_{w}\right)=\left(\frac{S_{w}-S_{\mathrm{wirr}}}{1-S_{\mathrm{wirr}}-S_{\mathrm{nwr}}}\right)^{n_{w}}, \\
& k_{r w}\left(S_{w}\right)=\left(\frac{S_{\mathrm{nw}}-S_{\mathrm{nwr}}}{1-S_{\mathrm{wirr}}-S_{\mathrm{nwr}}}\right)^{n_{\mathrm{nw}}},
\end{aligned}
$$

in which $S_{w}$ and $S_{\mathrm{nw}}$ are the non-wetting and wetting phase saturations, respectively, and $S_{\mathrm{wirr}}$ and $S_{\mathrm{nwr}}$ are the irreducible wetting and non-wetting phase saturations, respectively. $n_{w}$ and $n_{\mathrm{nw}}$ are the exponents for the wetting and non-wetting phases and both are set to 2 . The viscosity ratio between the phases and the porosity, $\phi$, are 1.0 and 0.2 , respectively. Table 1 reports the rest of the parameters for the different test cases in S.I. units except permeability, which is reported in millidarcy $(1 \mathrm{~m} D=9.869233 \times$ $10^{-16} \mathrm{~m}^{2}$ ).

The domain is initially saturated with the non-wetting phase at $\left(1-S_{\text {wirr }}\right)$. The wetting phase is injected through the left boundary, the right boundary is open and the other boundaries are closed. The pressure is set to 0 at the right boundary.

The mesh is adapted to minimise the error of the saturation field only, providing a precision of $1 \%$ for this field.

Table 1 Model set-up for the test cases $4.1-4.3 ; u_{\text {in }}$ is the inlet velocity

\begin{tabular}{llllllll}
\hline & $K_{1}$ & $K_{2}$ & $K_{3}$ & $S_{\text {wirr }}$ & $S_{\text {nwr }}$ & $u_{\text {in }}$ & Length \\
\hline 4.1 & 1.0 & 10 & N/A & 0.2 & 0.2 & 1.0 & 1.0 \\
4.2 & 1.0 & 1000. & N/A & 0.0 & 0.0 & $1 \times 10^{-5}$ & 0.6 \\
4.3 & 100. & 200. & 1000. & 0.2 & 0.2 & $1 \times 10^{-5}$ & 0.6
\end{tabular}

The Courant number of the experiments is the infinitum norm of the local Courant number of each $\mathrm{CV}$ :

$C_{n}=\max \left\{\frac{q_{\alpha i j} \delta t}{h_{\mathrm{ij}}}, \forall i \in 1, \ldots, \mathrm{CVs}, \forall j \in 1, \ldots, \mathrm{CVs}\right\}$

where $q_{\alpha i}$ is the Darcy velocity across CVs $i$ and $j$, and $h_{\mathrm{ij}}$ is the distance between centres of the CVs $i$ and $j$. In this way, the accumulated Courant number criterion of Fig. 5 is defined as follows:

$\mathrm{AC}_{n}=\sum C_{n}^{t}$,

where $t$ is the time level. When $\mathrm{AC}_{n}$ is above the threshold then the value is reset and the accumulation process starts again.

The runtimes of the simulations have been obtained running in serial and using an Intel i6600U.

\subsection{D high permeability inclusion in a low permeability region}

In this section, a 2D domain containing a high permeability inclusion is considered (see Fig. 6). A time-step size of $5 \times 10^{-3}$ is chosen so the Courant number is always above 10 to ensure that the shock-front moves out of the high resolution region of the mesh. Two experiments are compared: in one case, the mesh is adapted every time step using the algorithm described in Fig. 3. In the other case, the new algorithm described in Fig. 4 is used, and the mesh is adapted every time-step inside the non-linear solver.

Figure 7 shows the saturation front at four different time levels, using the conventional approach (left plots of each set) and the new approach adapting the mesh within the non-linear solver (right plots of each set). Figure $7 \mathrm{a}$ shows the initial mesh and the initial condition. Figure $7 \mathrm{~b}$ shows the saturation field at time-step 1 . The new approach has already caused the mesh to change, increasing the resolution at the shock-front, while the conventional

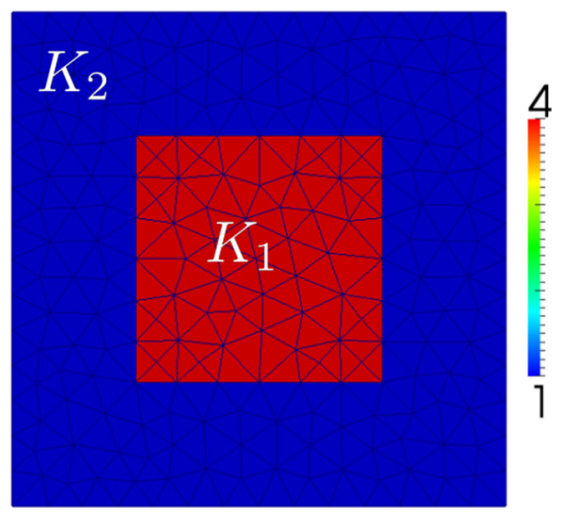

Fig. 6 Initial mesh and permeability map 

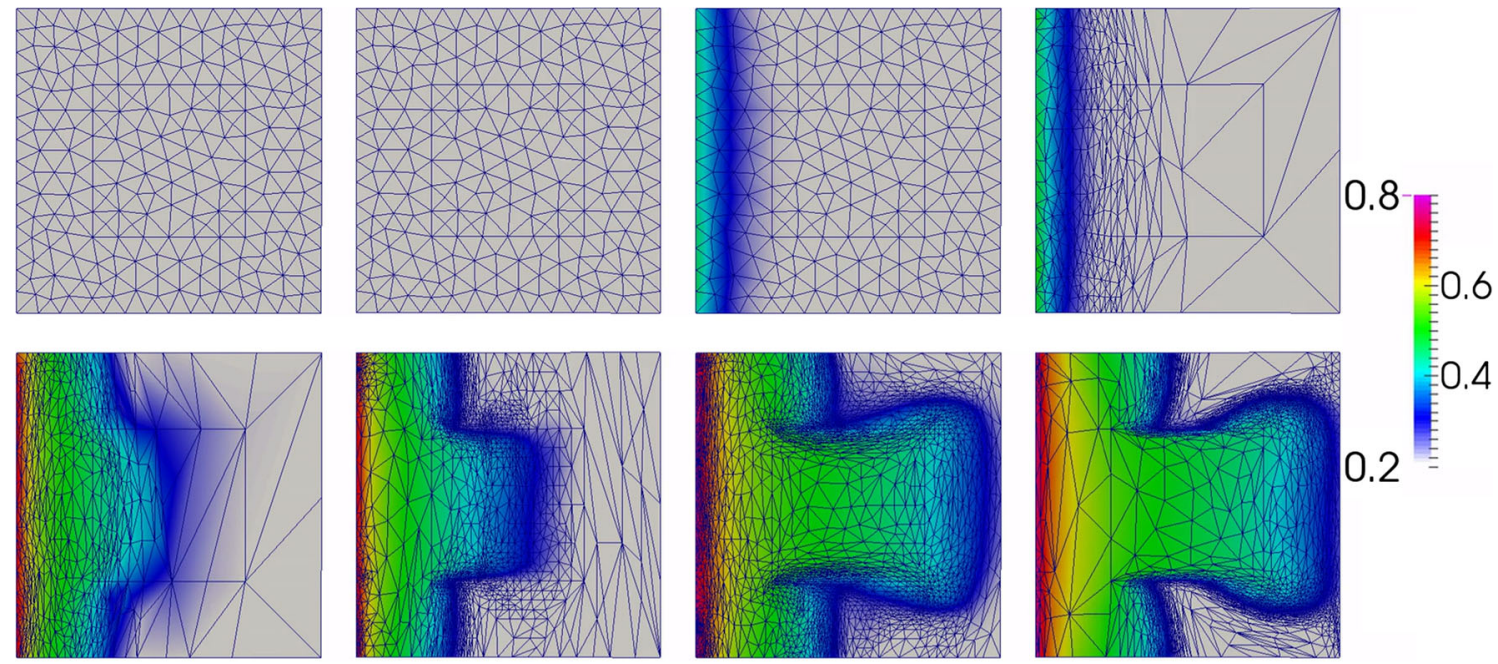

Fig. 7 Saturation maps at various time-steps comparing the conventional approach, adapting the mesh every time-step (left figure) to the new approach, adapting the mesh within the non-linear solver (right

approach is still using the initial mesh. Figure $7 \mathrm{c}$ shows the time level at which the wetting saturation has reached the highly permeable inclusion. The difference between the two adaptivity approaches is now more pronounced, because the velocity of the shock-front is increased in the higher permeability inclusion. The shock-front is dispersed in the conventional approach because it moves into a coarser region of the mesh. However, the new method accurately increases the resolution where necessary, keeping the shockfront well defined. Figure $7 \mathrm{~d}$ shows the time-level at which the injected wetting phase reaches the outlet boundary. Again, the new adaptivity method provides a better defined solution.

To assess the quality of the new method, a fine, fixed mesh case was run (27136 elements). The resolution of the fixed mesh is equivalent to the maximum resolution allowed in the DMO algorithm and the same time step was used. The new method of adapting the mesh within the non-linear solver provides results with almost the same quality as a fine fixed mesh (Fig. 8). Conversely, adapting the mesh every time-step produces a lower quality solution. Calculating the $L_{1}, L_{2}$ and $L_{\infty}$ norms of the error, considering the fine fixed mesh as the correct solution, shows that adapting the mesh within the non-linear solver yields an error 2.8, 3.3 and 5.7 times better than using standard DMO.

Figure 9a shows the number of non-linear iterations versus the time-step. Adapting the mesh within the nonlinear solver requires extra computational effort. However, the average extra cost is very low, as it requires around two extra non-linear iterations when compared to adapting the mesh every time-step, and one extra non-linear iteration when compared to a fine fixed mesh. Figure $9 \mathrm{~b}$ shows figure). a Initial state and mesh. b First time-step. c Time level at which the injected phase reaches the high permeability inclusion. d Time level at which the injected phase reaches the outlet boundary

how adapting the mesh within the non-linear solver initially introduces more elements. However, for this case, the new method also reduces the number of elements faster and earlier than adapting the mesh every time-step.

A final test is performed for this model. Now, the permeabilities are swapped, being low permeable in the interior square and high permeable the outer part. Moreover, the viscosity contrast in increased to 50 to further explore the behaviour of the presented method. Figure 10 shows the saturation maps when considering the new setup. It can be seen how the flow clearly goes around the low permeable inclusion. However, numerical dispersion from the high permeable region to the low permeable region appears. This is due to effect of the CVFEM formulation, where the CVs span different petrophysical materials ([14]) creating an artificial dispersion from the high permeable regions to the low permeable ones. Some approaches have

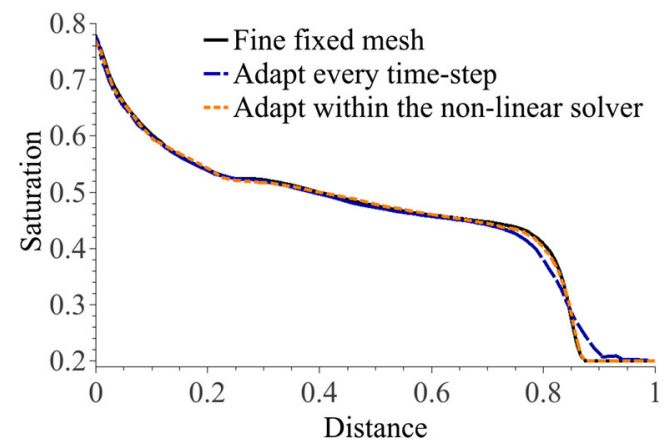

Fig. 8 1D saturation profile along the diagonal from top-left to bottom-right of Fig. 7d using a fine, fixed mesh, DMO every time-step and DMO within the non-linear solver 
Fig. 9 a Number of non-linear iterations per time-step for the first 18 time-steps for the test case 4.1 ; the runtime of this simulation is $220 \mathrm{~s}$. b Number of elements per time-step for the first 18 time-steps; the runtime of this simulation is $230 \mathrm{~s}$

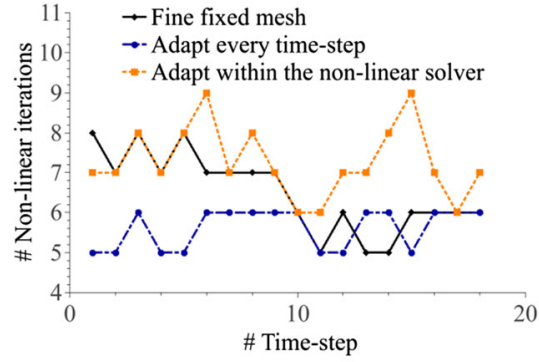

a

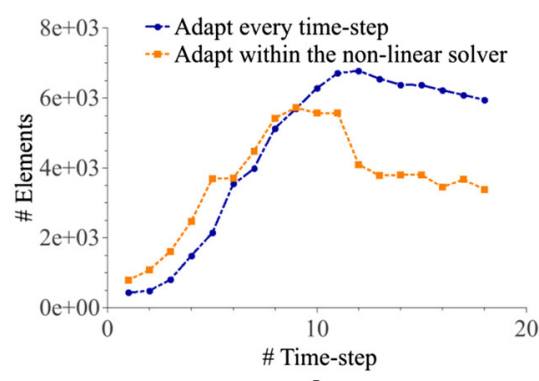

b been developed to try to solve this problem ([1, 6, 34, $35,41]$ ); here, the dispersion is reduced by using DMO. The effect of the high viscosity appears with a less sharp leading shock-front. However, the method proposed is able to properly capture it by placing the mesh resolution where required, Fig. 10a. The DMO algorithm clearly detects that high resolution is required not only at the shock-front but more importantly around the low permeable object to reduce the artificial dispersion from the high permeable area to the low permeable region, Fig. 10b, c and d.

\subsection{D high permeabilty fractures in a low permeable region}

The model considered in this section is a subset of fractures taken from [7]. The fractures have high permeability (see Table 1), and very high aspect ratio (width of $3 \mathrm{~mm}$ ). The wetting phase is injected into the model over the left boundary and only the right boundary is open (see Fig. 11). The flow moves considerably faster through the fractures. The time-step size used in this case is set to $10^{3}$ and the high permeability contrasts result in maximum Courant numbers up to 500 .

Figure 12a, c shows the saturation field and the mesh at two different time-levels, adapting the mesh conventionally every time-step. Figure $12 \mathrm{~b}, \mathrm{~d}$ shows the saturation field at the same time-levels but adapting the mesh within the non-linear solver using the new approach. Due to the large change in shock-front velocity when reaching the high-permeability fractures, adapting the mesh every timestep is not sufficient to correctly track the shock-front, generating regions with high artificial diffusion. This effect is particularly clear in Fig. 12a, in contrast to Fig. 12b, where it is significantly reduced by the new method.

For this numerical experiment, around one additional non-linear iteration is required in order to achieve convergence when adapting the mesh within the non-linear solver, (Fig. 13a). As in the previous test case, Fig. 13b shows that adapting the mesh within the non-linear solver initially introduces more elements, but later the number of elements is reduced earlier and faster than adapting the mesh every time-step. To further explore the costs associated with the proposed approach, this simulation is also run with mesh adaptivity and a gradation parameter, such as the high resolution of the mesh would cover the shock-front at the next time level. The number of elements required in the second time step (because in the initial time the mesh is the given one) is above 260000 , which is one order of magnitude bigger that the required amount of elements using the presented approach.

In this case, the difference in Courant number between the two experiments using the conventional and new adaptivity methods is larger, as the new method is able to introduce higher resolution. When adapting every time-step, there is more artificial diffusion (see Fig. 12a, b), so the DMO algorithm introduces less resolution as the saturation field is more diffusive, i.e. the shock-front is less sharp. Consequently, to achieve converge using the new method,

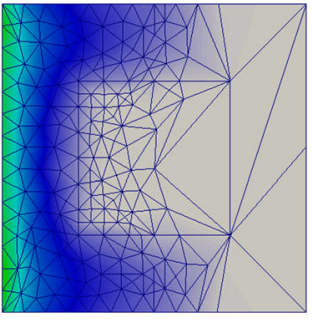

a

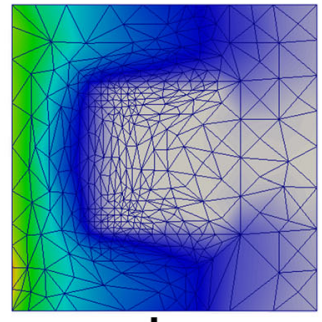

b

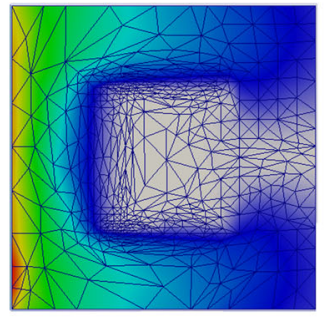

C

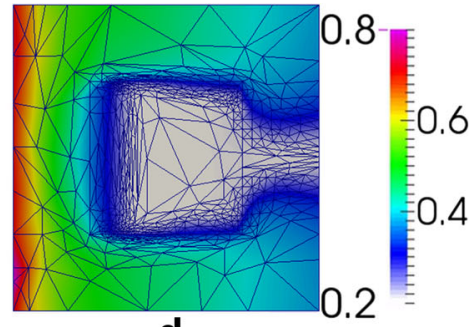

d
Fig. 10 Saturation maps at various time-steps showing the effect of a low permeable inclusion and high viscosity ratio. a Initial state and mesh. b First time-step. c Time level at which the injected phase clearly reaches the outlet boundary. $\mathbf{d}$ Time level at which the injected phase almost surrounds the low permeable inclusion 


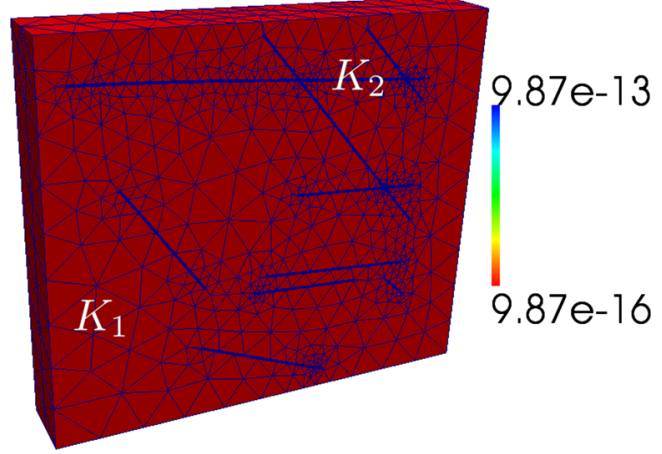

Fig. 11 Initial mesh and permeability map

more effort is required in the non-linear solver, i.e. more iterations are required. As an example, the Courant number when adapting the mesh within the non-linear solver can be up to four times bigger than when adapting every time-step.

\subsection{D fluvial channels}

In this numerical experiment, a set of fluvial channelfill sandbodies are considered, embedded in a nonreservoir background. Figure 14 shows the permeability map, dimensions and initial mesh of the computational domain. The non-reservoir background has been set to be transparent. The domain is characterised by an aspect ratio of 1 : 50. The wetting phase is injected over the right boundary and the left boundary is the only open boundary. The time-step size is set to $10^{6}$, resulting in Courant numbers above 300 .
Figure 15 shows the saturation field and the mesh at two different time-steps. Figure 15a, $\mathrm{c}$ is obtained adapting the mesh every time-step. Figure $15 b$, $d$ is obtained using the new method of adapting the mesh within the non-linear solver. The results obtained using the new method show a better defined shock-front than adapting the mesh every time-step, as the standard DMO cannot correctly place the high resolution of the mesh where required.

For this numerical experiment, an average of four more non-linear iterations are required in order to achieve convergence when adapting the mesh within the non-linear solver (see Fig. 16a). Also, Fig. 16b shows that, adapting the mesh within the non-linear solver requires, for this experiment, more elements.

In this case, the difference in Courant numbers between the conventional and new methods is very important. The Courant number is $30 \%$ bigger when adapting the mesh within the non-linear solver (Courant number of 500 instead of 350 when using standard DMO). This difference comes from the fact that when using the standard DMO, more numerical diffusion is introduced, so a coarser mesh is sufficient to represent the solution field. It is important to note that the method presented here is not necessarily intended to be used at the extreme case when the mesh needs to be adapted every time-step. In this numerical experiment for example, adapting the mesh within the non-linear solver with a lower time-step size would not require so much extracost compared to standard DMO. In that case, unless sudden changes in the Courant number, the extra-cost compared with standard DMO would be smaller as the meshes would be similar (for example with Courant numbers of around 10). However, if a sudden increase in the velocity of the
Fig. 12 2D slice through the 3D saturation field at different time-steps comparing adapting the mesh every time-step vs. adapting the mesh within the non-linear solver. a Saturation field after two time-steps adapting the mesh every timestep. b Saturation field after two time-steps adapting the mesh within the non-linear solver. c Saturation field after four timesteps adapting the mesh every time-step. d Saturation field after four time-steps adapting the mesh within the non-linear solver. It can be seen how, as the flow moves much faster through the high-permeable fractures, Figs. (a) and (c) suffer from more artificial diffusion than (b) and (d)

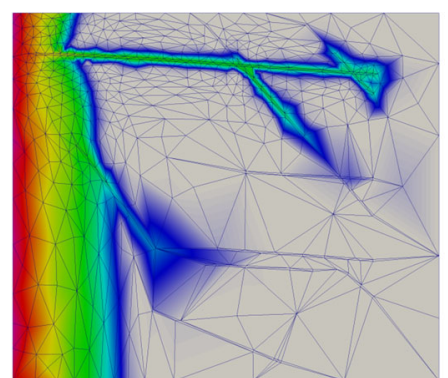

a

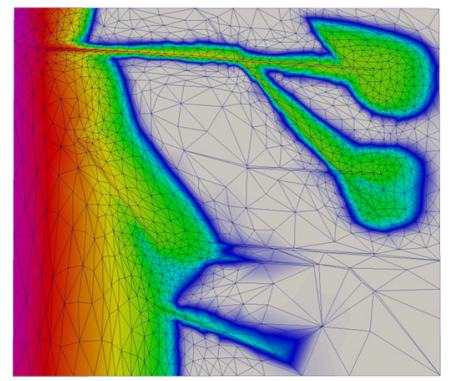

C

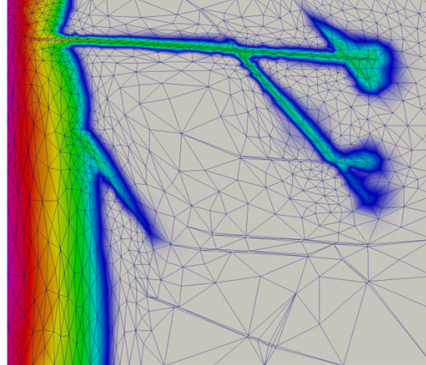

b

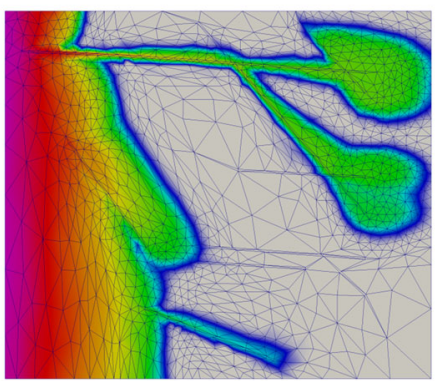

d 
Fig. 13 a Number of non-linear iterations per time-step for the first 20 time-steps for the test case 4.2; the runtime of this simulation is 55 minutes. $\mathbf{b}$ Number of elements per time-step for the first 20 time-steps 4.2 ; the runtime of this simulation is 62 minutes

Fig. 14 Initial mesh, permeability map and a crosssection showing the intersection between different channels

Fig. 15 Top view of the saturation field at different time-steps comparing the conventional approach of adapting the mesh every time-step vs. the new method of adapting the mesh within the non-linear solver. a Saturation field after one time-step using the conventional approach. b Saturation field after one time-step using the new method. c Saturation field after five timesteps using the conventional approach. d Saturation field after five time-steps using the new method. It can be seen from figures (a) and (c) how the shock-front in the small channels is not captured by the conventional approach as it reaches the end of the domain before the mesh is adapted

Fig. 16 a Number of non-linear iterations per time-step for the first 20 time-steps for the test case 4.3 ; the runtime of this simulation is 32 minutes. b Number of elements per time-step for the first 20 time-steps ; the runtime of this simulation is $67 \mathrm{~min}$

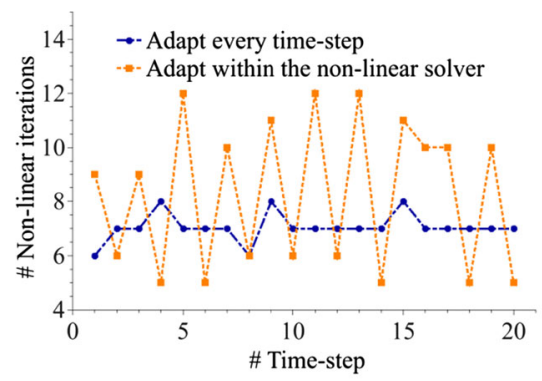

a

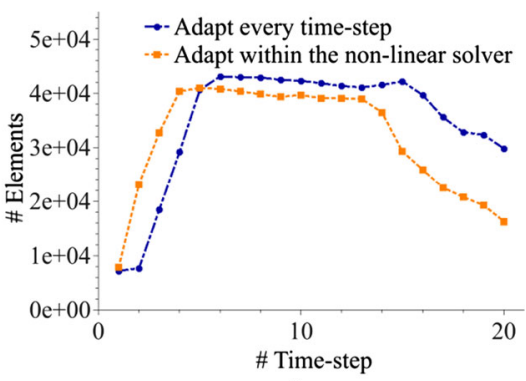

b

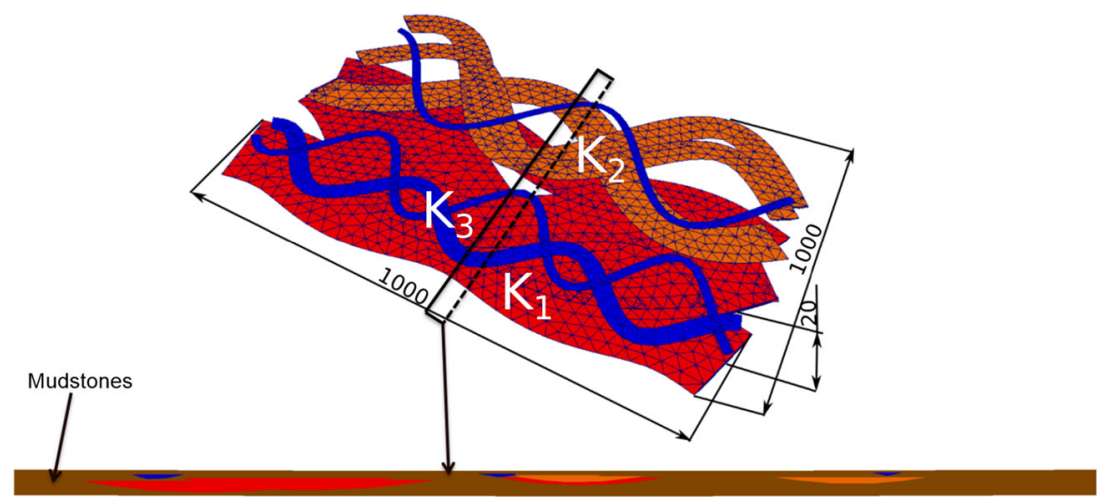

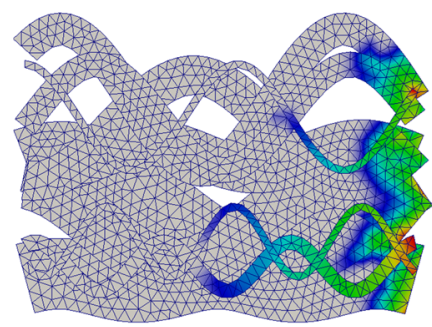

a

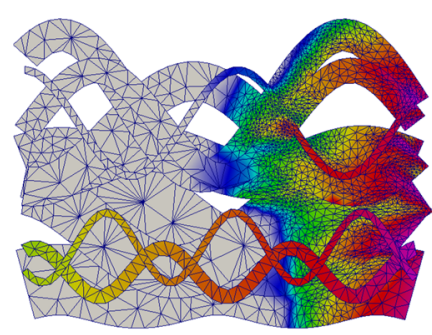

c

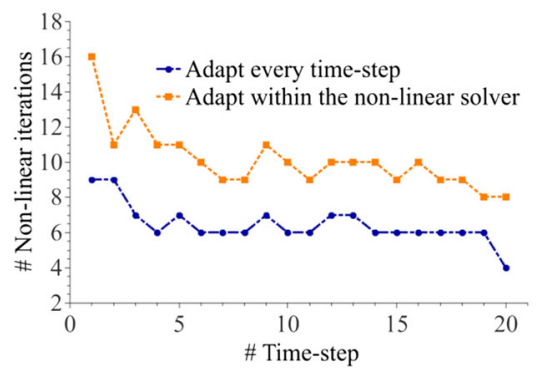

a

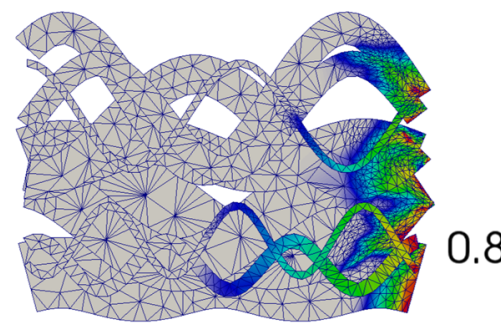

b

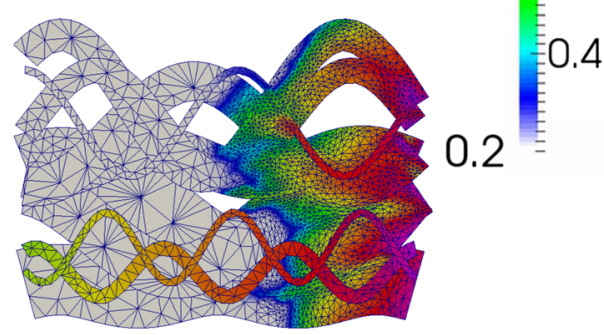

d

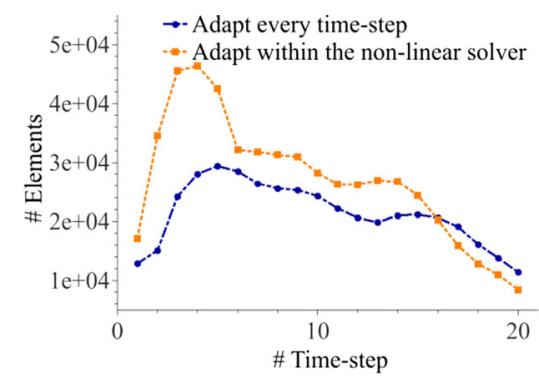

b 


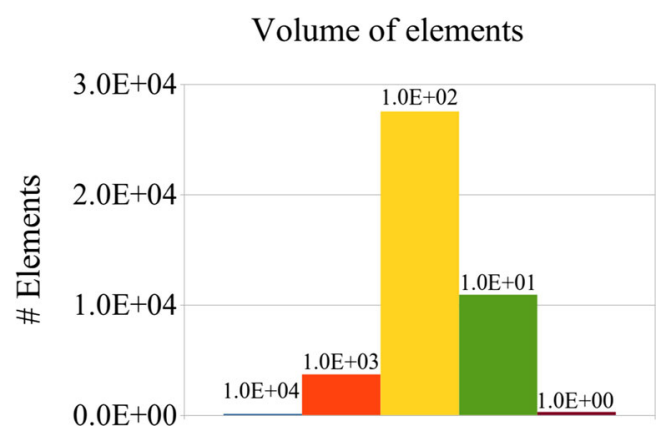

Fig. 17 Number of elements with a certain volume (same order of magnitude) for the case shown in Fig. 15d

shock-front occurs (due to a high permeability jump, for example), the standard DMO would be cheaper but would provide a more diffusive solution. However, adapting within the non-linear solver, despite requiring an extra-cost, would be able to keep a higher quality of the solution, and continue the simulation without a significant loss of quality in the solution.

For this test case, a histogram of element volumes is presented to show the variability of element sizes when dealing with unstructured meshes and DMO. Figure 17 shows the number of elements grouped with the same order of magnitude of volume for the case shown in Fig. 15d.

The element sizes span four orders of magnitude. This shows the importance of being able to adapt the mesh for high Courant numbers. As the mesh is modified, it becomes part of model as it introduces an important variation on the local Courant numbers throughout the domain.

\section{Conclusions}

A new methodology to accurately place high resolution when using AMR/DMO for multiphase porous media flow with high Courant numbers is presented. This method estimates where the high resolution mesh is required for the field of interest and the mesh is adapted accordingly. For this, extra non-linear iterations may be required compared to using a fixed mesh. However, the extra cost is not too high (typically a few extra non-linear iterations under the same circumstances) and results in solutions with less numerical diffusion. The presented method enables the use of large Courant numbers ( $>10$ and tested up to 500) together with $\mathrm{AMR} / \mathrm{DMO}$ and is independent of the discretisation scheme used, being tested here using CVFEM and DMO.

Such high Courant numbers may not be desired by the user, but may arise as a consequence of the flow behaviour induced by anisotropic meshes and/or heterogeneous material properties when using fixed time-step sizes or time-step sizes controlled by the performance of the nonlinear solver. The presented method can be considered as an extra-step to obtain a more robust and accurate alternative to standard mesh optimisation routines (AMR/DMO) for multiphase porous media flow.

Acknowledgements Funding for Salinas from ExxonMobil is gratefully acknowledged. Pavlidis would like to acknowledge the support from: EPSRC "Reactor Core-Structure Relocation Modelling for Severe Nuclear Accident" and Horizon 2020 "In-Vessel Melt Retention". Xie is supported by EPSRC ('Multi-Scale Exploration of Multiphase Physics in Flows'-MEMPHIS). Part funding for Jackson under the TOTAL Chairs programme at Imperial College is also acknowledged. We thank Dr. Melnikova for providing the domain used in Section 4.3. No data was generated in the course of this work. For further information, please contact the corresponding author at (pablo.salinas@imperial.ac.uk), the AMCG Group (www.imperial.ac.uk/earth-science/research/research-groups/ amcg/) or the NORMS group (www.imperial.ac.uk/earth-science/ research/research-groups/norms) as required.

Open Access This article is distributed under the terms of the Creative Commons Attribution 4.0 International License (http:// creativecommons.org/licenses/by/4.0/), which permits unrestricted use, distribution, and reproduction in any medium, provided you give appropriate credit to the original author(s) and the source, provide a link to the Creative Commons license, and indicate if changes were made.

\section{References}

1. Abushaikha, A., Blunt, M., Gosselin, O., Pain, C., Jackson, M.D.: Interface control volume finite element method for modelling multi-phase fluid flow in highly heterogeneous and fractured reservoirs. Journal of Computational Physics pp. 41-61. https://doi.org/10.1016/j.jcp.2015.05.024 (2015)

2. Adam, A., Pavlidis, D., Percival, J., Salinas, P., Xie, Z., Fang, F., Pain, C., Muggeridge, A., Jackson, M.D.: Higherorder conservative interpolation between control-volume meshes: application to advection and multiphase flow problems with dynamic mesh adaptivity. J. Comput. Phys. 321, 512-531 (2016). https://doi.org/10.1016/j.jcp.2016.05.058

3. Alauzet, F., Frey, P., George, P., Mohammadi, B.: 3d transient fixed point mesh adaptation for time-dependent problems: application to cfd simulations. J. Comput. Phys. 222(2), 592-623 (2007). https://doi.org/10.1016/j.jcp.2006.08.012

4. Alauzet, F., Loseille, A.: A decade of progress on anisotropic mesh adaptation for computational fluid dynamics. Comput. Aided Des. 72, 13-39 (2016). https://doi.org/10.1016/j.cad.2015.09.005. 23rd International Meshing Roundtable Special Issue: Advances in Mesh Generation

5. Anderson, D.G.: Iterative procedures for nonlinear integral equations. J. Assoc. Comput. Machinery 12, 547-560 (1965)

6. Bazr-Afkan, S., Matthäi, S.: A new hybrid simulation method for multiphase flow on unstructured grids with discrete representation of material interfaces. MIAMG pp. 294-305 (2011)

7. Belayneh, M., Geiger, M., Matthai, S.: Numerical simulation of water injection into layered fractured carbonate reservoir analogs. AAPG Bull. 90, 1473-1493 (2006)

8. Brooks, R., Corey, A.: Hydrology papers, vol. 3. Hydraulic properties of porous media Colorado State University, Chap (1964) 
9. Dahle, H.K., Espedal, M., Ewing, R.E., Saevereid, O.: Characteristic, local grid refinement techniques for reservoir flow problems. International Journal of Numerical Engineering 34, 1051 (1992)

10. Dahle, H.K., Espedal, M.S., Ewing, R.E., Sævereid, O.: Characteristic adaptive subdomain methods for reservoir flow problems. Numerical Methods for Partial Differential Equations 6(4), 279309 (1990). https://doi.org/10.1002/num.1690060402

11. Debbabi, Y., Jackson, M.D., Hampson, G.J., Fitch, P.J.R., Salinas, P.: Viscous crossflow in layered porous media. Transport in Porous Media 117(2), 281-309 (2017). https://doi.org/10.1007/s11242-017-0834-z

12. Denver, L.E., Phillips, D.C.: Stratigraphic geocellular modeling. Geobyte 5, 45-47 (1990)

13. Deveugle, P., Jackson, M.D., Hampson, G., Farrell, M., Sprague, A., Stewart, J., Calvert, C.: Characterization of stratigraphic architecture and its impact on fluid flow in a fluvialdominated deltaic reservoir analog: upper cretaceous ferron sandstone member, utah. AAPG Bull. 95, 693-727 (2011). https://doi.org/10.1306/09271010025

14. Edwards, M.: Higher-resolution hyperbolic-coupled-elliptic fluxcontinuous CVD schemes on structured and unstructured grids in 2-D. Int. J. Numer. Meth. Fluids 51, 1059-1077 (2006)

15. Edwards, M., Christie, M.: Dynamically adaptive Godunov schemes with renormalization in reservoir simulation. Society of Petroleum Engineers (1993)

16. Faigle, B., Helmig, R., Aavatsmark, I., Flemisch, B.: Efficient multiphysics modelling with adaptive grid refinement using a MPFA method. Comput. Geosci. 18(5), 625-636 (2014). https://doi.org/10.1007/s10596-014-9407-1

17. Farrell, P., Maddison, J.: Conservative interpolation between volume meshes by local Galerkin projection. Comput. Methods Appl. Mech. Eng. 200(1-4), 89-100 (2011). https://doi.org/10.1016/j. cma.2010.07.015

18. Forsyth, P.: A control volume, finite element method for local mesh refinement in thermal reservoir simulation. SPE Reserv. Eng. 5(4), 561-566 (1990)

19. Geiger, S., Roberts, S., Matthäi, S., Zoppou, C., Burri, A.A.: Combining finite element and finite volume methods for efficient multiphase flow simulations in highly heterogeneous and structurally complex geologic media. Geofluids 4(4), 284-299 (2004)

20. Gomes, J.L.M.A., Pavlidis, D., Salinas, P., Xie, Z., Percival, J.R., Melnikova, Y., Pain, C.C., Jackson, M.D.: A force-balanced control volume finite element method for multi-phase porous media flow modelling. Int. J. Numer. Meth. Fluids 83, 431-445 (2017). https://doi.org/10.1002/fld.4275

21. Graham, G., Jackson, M.D., Hampson, G.: Three-dimensional modeling of clinoforms in shallow-marine reservoirs: part 1. concepts and application. AAPG Bull. 99, 1013-1047 (2015). https://doi.org/10.1306/01191513190

22. Hiester, H., Piggott, M., Allison, P.: The impact of mesh adaptivity on the gravity current front speed in a two-dimensional lockexchange. Ocean Model. 38(1-2), 1-21 (2011). https://doi.org/10. 1016/j.ocemod.2011.01.003

23. Hornung, R.D., Trangenstein, J.A.: Adaptive mesh refinement and multilevel iteration for flow in porous media. J. Comput. Phys. 136(2), 522-545 (1997). https://doi.org/10.1006/jcph.1997.5779

24. Jackson, M.D., Percival, J., Mostaghimi, P., Tollit, B., Pavlidis, D., Gomes, J., El-Sheikh, A., Salinas, P., Muggeridge, A., Blunt, M.: Reservoir modeling for flow simulation by use of surfaces, adaptive unstructured meshes, and an overlapping-control-volume finite-element method. SPE Reserv. Eval. Eng. 18(2), (2015). https://doi.org/10.2118/163633-PA

25. Janković, I., Fiori, A., Dagan, G.: Modeling flow and transport in highly heterogeneous three-dimensional aquifers: ergodicity, Gaussianity, and anomalous behavior-1. Conceptual issues and numerical simulations. Water Resour. Res. 42(SUPPL.), 1-9 (2006). https://doi.org/10.1029/2005WR004734

26. Jenny, P., Tchelepi, H., Lee, S.: Unconditionally convergent nonlinear solver for hyperbolic conservation laws with S-shaped flux functions. J. Comput. Phys. 228, 7497-7512 (2009)

27. Lee, S., Wheeler, M., Wick, T.: Pressure and fluid-driven fracture propagation in porous media using an adaptive finite element phase field model. Comput. Methods Appl. Mech. Eng. 305, 111-132 (2016). https://doi.org/10.1016/j.cma.2016.02.037

28. Li, B., Tchelepi, H.A.: Nonlinear analysis of multiphase transport in porous media in the presence of viscous, buoyancy, and capillary forces. J. Comput. Phys. 297, 104-131 (2015). https://doi.org/10.1016/j.jcp.2015.04.057

29. MacDonald, A.C., Falt, L.M., Hektoen, A.L.: Stochastic modeling of incised valley geometries. Am. Assoc. Pet. Geol. Bull. 82, 1156-1172 (1998)

30. Matthai, S., Mezentsev, A., Belayneh, M.: Control-volume finite element two-phase flow experiments with fractured rock represented by unstructured 3D hybrid meshes. SPE 93341 (2005)

31. Matthai, S., Mezentsev, A., Belayneh, M.: Finite elementnode centered finite-volume two-phase flow experiments with fractured rock represented by unstructured hybrid element meshes. SPE Reserv. Eval. Eng. 10(6), (2007). https://doi.org/10.2118/ 93341-PA

32. Mostaghimi, P., Percival, J., Pavlidis, D., Ferrier, R., Gomes, J., Gorman, G., Jackson, M.D., Neethling, S., Pain, C.: Anisotropic mesh adaptivity and control volume finite element methods for nucmerical simulation of multiphase flow in porous media. Math. Geosci. 347, 673-676 (2015)

33. Natvig, J.R., Lie, K.: Fast computation of multiphase flow in porous media by implicit discontinuous Galerkin schemes with optimal ordering of elements. Journal of Computational physics. https://doi.org/10.1016/j.jcp.2008.08.024 (2008)

34. Nick, H., Matthai, S.: Comparison of three FE-FV numerical schemes for single- and tw-phase flow simulation of fractured porous media. Transp. Porous Med 90, 421-444 (2011)

35. Nick, H., Matthai, S.: A hybrid finite-element finite-volume method with embedded discontinuities for solute transport in heterogeneous media. Vadose Zone J. 10, 299-312 (2011)

36. Osman, H., Graham, G., Moncorge, A., Jackson, M.D.: Do we need to include short length-scale variability in reservoir models? In: EAGE annual conference (2018)

37. Pain, C., Umpleby, A., de Oliveira, C., Goddard, A.: Tetrahedral mesh optimisation and adaptivity for steady-state and transient finite element calculations. Comput. Methods Appl. Mech. Eng. 190(29), 3771-3796 (2001). https://doi.org/10.1016 /S0045-7825(00)00294-2

38. Pyrcz, M.J., Catuneau, O., Deutch, C.V.: Stochastic surface-based modeling of turbidite lobes. Am. Assoc. Pet. Geol. Bull. 89, 177-191 (2005)

39. Salinas, P., Pavlidis, D., Xie, Z., Adam, A., Pain, C.C., Jackson, M.D.: Improving the convergence behaviour of a fixed-point-iteration solver for multiphase flow in porous media. International Journal for Numerical Methods in Fluids. https://doi.org/10.1002/fld.4357 (2016)

40. Salinas, P., Pavlidis, D., Xie, Z., Jacquemyn, C., Melnikova, Y., Pain, C.C., Jackson, M.D.: Improving the robustness of the control volume finite element method with application to multiphase porous media flow. Int. J. Numer. Methods Fluids 0, 1-12 (2017). https://doi.org/10.1002/fld.4381

41. Salinas, P., Pavlidis, D., Xie, Z., Osman, H., Pain, C., Jackson, M.: A discontinuous control volume finite element method for multiphase flow in heterogeneous porous media. J. Comput. Phys. 352, 602-614 (2018). https://doi.org/10.1016/j.jcp.2017.09.058 
42. Sammon, P.: Dynamic grid refinement and amalgamation for compositional simulation. Society of Petroleum Engineers (2003)

43. Schmidt, G., Jacobs, F.: Adaptive local grid refinement and multigrid in numerical reservoir simulation. J. Comput. Phys. 77, 140-165 (1988)

44. Solano, J.M.S., Jackson, M.D., Sparks, R.S.J., Blundy, J.: Evolution of major and trace element composition during melt migration through crystalline mush: implications for chemical differentiation in the crust. Am. J. Sci. 314, 895-939 (2014). https://doi.org/10.2475/05.2014.01

45. Soltanian, M.R., Amooie, M.A., Cole, D.R., Graham, D.E., Hosseini, S.A., Hovorka, S., Pfiffner, S.M., Phelps, T.J., Moortgat, J.: Simulating the Cranfield geological carbon sequestration project with high-resolution static models and an accurate equation of state. Int. J. Greenhouse Gas Control 54(Part 1), 282-296 (2016). https://doi.org/10.1016/j.ijggc.2016.10.002

46. Sterck, H., Ullrich, P.: Introduction to computational mathematics. University of Waterloo (2006)

47. Trangenstein, J.A.: Multi-scale iterative techniques and adaptive mesh refinement for flow in porous media. Adv. Water Resour. 25, 1175-1213 (2002)

48. Xie, Z., Pavlidis, D., Percival, J., Gomes, J., Pain, C., Matar, O.: Adaptive unstructured mesh modelling of multiphase flows. Int. J. Multiphase Flow 67, 104-110 (2014) 